FACTA UNIVERSITATIS

Series: Mechanical Engineering Vol. 19, No 4, 2021, pp. 657 - 680

https://doi.org/10.22190/FUME201009045S

Original scientific paper

\title{
FOURTH-ORDER STRAIN GRADIENT BAR-SUBSTRATE MODEL WITH NONLOCAL AND SURFACE EFFECTS FOR THE ANALYSIS OF NANOWIRES EMBEDDED IN SUBSTRATE MEDIA
}

\section{Worathep Sae-Long ${ }^{1}$, Suchart Limkatanyu², Piti Sukontasukkul ${ }^{3}$, Nattapong Damrongwiriyanupap ${ }^{1}$, Jaroon Rungamornrat ${ }^{4}$, Woraphot Prachasaree ${ }^{2}$}

${ }^{1}$ Civil Engineering Program, School of Engineering, University of Phayao, Thailand

${ }^{2}$ Dept. of Civil Eng., Faculty of Engineering, Prince of Songkla University, Thailand ${ }^{3}$ Construction and Building Materials Research Center, Department of Civil Engineering, King Mongkut's University of Technology North Bangkok, Thailand

${ }^{4}$ Applied Mechanics and Structures Research Unit, Department of Civil Engineering, Faculty of Engineering, Chulalongkorn University, Bangkok, Thailand

\begin{abstract}
This paper presents a new analytical bar-substrate model for the analysis of an isotropic and homogeneous nanowire embedded in an elastic substrate. A fourth-order nonlocal strain gradient model based on a thermodynamic approach is employed to represent the small-scale effect (nonlocal effect) while the Gurtin-Murdoch continuum model based on the surface elastic theory is used to account for the size-dependent effect (surface energy effect). The proposed model is derived from the virtual displacement principle, leading to the governing differential equations and its associated natural boundary conditions. The analytical solutions of the sixth-order governing differential equation for the nanowire-substrate element are provided, and were employed in numerical simulations. Two numerical simulations are used to demonstrate the performance and to investigate the characteristics of the fourth-order nonlocal strain gradient model on nanowire responses, when compared to the classical model and the second-order nonlocal strain gradient model. The first simulation investigates the influences of nonlocal and surface effects on the responses of a nanowire embedded in an elastic substrate, while the second simulation study assessed the sensitivity of system stiffness on parameters in the nanowire-substrate model.
\end{abstract}

Key words: Nanobeam-Substrate Model, Displacement-Based Formulation, Nonlocal Thermodynamic Approach, Fourth-Order Nonlocal Strain Gradient Model, Nonlocal Strain Gradient

Received October 09, 2020 / Accepted December 07, 2020

Corresponding author: Suchart Limkatanyu

Department of Civil Engineering, Faculty of Engineering, Prince of Songkla University, Songkhla, 90112, Thailand

E-mail: suchart.1@psu.ac.th 


\section{INTRODUCTION}

With the rapid development of technology and science, nanotechnology plays a greater role in several applications and has led to a huge effort to study nano-sized structures in the engineering fields. However, the characteristics of these nanoscale structures differ from the large-scale structures, and size or scale affects those behaviors [1-5]. Furthermore, the development of numerical models for replacing the results from the experiments, which are expensive and require specific equipment for testing or observation, is a challenging problem to the structural analysis community, motivating also this current study. The available numerical models to characterize the mechanical responses of nanostructures in the structural engineering field fall into two categories, namely, (i) atomistic models; and (ii) continuummechanics models. The atomistic models are established at molecular or atomic level based on several techniques, such as molecular dynamics simulations [6-7], density functional theory [8-9], etc. These models can provide comprehensive details and are beneficial in the analysis of small and simple nanostructures [10]. However, the atomistic simulations are very cumbersome and have high computational costs when applied to complex or large nanostructure systems. For large-scale nanostructures, the continuum-mechanics models are an attractive alternative to simulate the mechanical characteristics of the nanostructures, with good applicability and simplicity. Although the classical continuum models do not originally incorporate size-scale effect, this model type can be applied with non-classical continuum theories, and have been named "nonlocal" continuum models [11-12].

According to the nonlocal continuum models, several non-classical continuum theories are employed to account for the size-scale or the so-called "nonlocal" effect, which introduces additional material length-scale parameters in nonlocal elasticity theory [13-14], couple stress theory [15], modified couple stress theory [16-17], strain gradient theory [18-19], or in modified strain gradient [20-21]. In the last twenty years, the nonlocal elasticity theory of Eringen [13-14] was the most popular for addressing sizescale effect, and was widely applied in several studies of nanostructures [22-24]. However, there are paradoxical results (the vanishing of nonlocal effect) in the modeled responses of nanostructures, such as for a cantilever beam subjected to an end load [2526]. Romano et al. [27] reported that this paradox is caused by the incompatibility of equilibrium equations and constitutive boundary terms. To avoid the ill-posedness of Eringen nonlocal elasticity theory, Peddieson et al. [25] suggested the nonlocal strain gradient theory as expanded from the general integral constitutive equation of Eringen nonlocal elasticity theory [14]. When only the first two terms were considered, the general form of the stress-strain relation based on the nonlocal strain gradient theory was similar to the form of the simplified strain-gradient elasticity model as proposed Altan and Aifantis [28]. This led to the so-called "second-order" nonlocal strain gradient model [29-30]. The performance of this nonlocal constitutive model has been investigated by several researchers [23, 29, 31-37]. For example, Zaera et al. [31] studied the inconsistency of the strain gradient model with the boundary terms. Khodabakhshi and Reddy [23] presented the governing equations and boundary terms of the strain gradient beam model based on von Karman nonlinearity in the Euler-Bernoulli beam theory, Timoshenko beam theory, and thirdorder Reddy beam theory. Kong et al. [32] used the strain gradient theory to investigate static and dynamic characteristics of Euler-Bernoulli beams. Barretta and Marotti de Sciarra [33] proposed a strain gradient constitutive model based on a consistent thermodynamic approach for the analysis of carbon nanotubes (CNTs). This concept was later applied to 
establish the modified constitutive laws for Euler-Bernoulli nanobeams [34-35], Timoshenko nanobeams [36], and a nanowire [37]. Narendar and Gopalakrishnan [29] studied an ultrasonic wave dispersion characteristics of a nanorod based on nonlocal strain gradient model. These studies have confirmed the superiority of the strain gradient model. In this work, the nonlocal strain gradient model based on the thermodynamic framework is chosen to improve the proposed model and to eliminate the paradoxical results.

The size-dependent effect or the so-called "surface energy" is caused by altered energy of the atoms at a free surface. This effect often plays a greater role in the mechanical responses of small-scale structures, when the surface-to-volume ratio is large, as found in several publications [38-41]. To incorporate the surface energy within the continuum model, Gurtin and Murdoch [42-43] proposed a surface elasticity theory and established a surface continuum model for isotropic materials based on zero thickness of the surface layer. To confront the surface effect, a lot of literature [24, 44-48] has applied the Gurtin and Murdoch surface model to nanostructures. For example, Liu and Rajapakse [44] studied the static and dynamic characteristics of elastic nanobeams under different load conditions. Fu et al. [45] later extended the work of Liu and Rajapakse [44] to cover the nonlinear analysis of both static and dynamic nanobeam problems. Limkatanyu et al. [24] investigated the flexural responses of nanobeams based on the Eringen nonlocal elasticity theory, while Fu et al. [46] studied these responses based on the strain gradient theory. Furthermore, Lim and He [47] examined the buckling behavior of a micro/nanofilm in relation to the nonlinear buckling characteristics of cylindrical nanoshells based on the classical shell theory proposed by Sahmani et al. [48]. In this current work, the Gurtin-Murdoch model [42-43] is applied to represent the surface surrounding effect within a uniaxial member in the nanoscale.

Among the several continuum models for the analysis of a nanowire embedded in a substrate, many approaches were used to establish the nanobar-substrate models. For example, Limkatanyu et al. [49-50] proposed nonlocal bar-substrate elements based on the nonlocal elasticity theory of Eringen [13-14] and the surface continuum model of Gurtin and Murdoch [42-43] for both displacement-based formulation [49] and forcebased formulation [50]. However, these models are ill-posed for the nanowire system under uniform load when the substrate modulus reaches zero [37]. Later, Sae-Long et al. [37] extended the strain gradient bar model based on the thermodynamic approach of Barretta and Marotti de Sciarra [33] to the bar-substrate model. This model can overcome the ill-posedness in a series of Eringen nonlocal models, but this model is categorized into the second-order nonlocal strain gradient model when only the first two terms of the nonlocal constitutive relation are considered.

In light of the need to upgrade the bar model for nano-scale problems, the main objective of this work was to develop the bar-substrate model with inclusion of higherorder terms in the nonlocal strain gradient model [25, 29] and the surface energy effect. The first three terms of the nonlocal strain gradient constitutive relation are considered, making this a "fourth-order" nonlocal strain gradient model [29-30]. This kind of model has been confirmed the superiority of performance to represent the complicated characteristics [51]. Thus, this work takes an interest in the influence of adding the third term in the nonlocal strain gradient model on the responses of the nanowire-substrate system, when compared to the second-order nonlocal strain gradient nanowire-substrate model [37]. The so-called "exact" displacement shape functions of the proposed sixthorder governing differential equilibrium equation given in this work are adapted from the 
analytical solutions of the beam model on Kerr-type foundation, proposed by Morfidis [52] and Avramidis and Morfidis [53]. To the best knowledge of the authors, this is the first time that the fourth-order nonlocal strain gradient model is applied in a displacementbased bar element to study the characteristics of a nanowire embedded in an elastic substrate.

The contents of this paper are organized as follows. Sections 2 and 3 provide the brief discussions of the nonlocal strain gradient model and the surface elastic theory that are employed to represent nonlocal and surface energy effects in this study, respectively. Then, the model formulation is presented in Section 4. It includes the governing differential equations such as the compatibility equations, constitutive relations, and equilibrium equation. Based on the virtual displacement principle, the governing differential equilibrium equation, its associated natural boundary conditions, and analytical solutions of the proposed model are also presented in this section. Next, Section 5 shows two numerical simulations of the characteristics of a nanowire embedded in a substrate. The first simulation is used to investigate the higher-order term in the fourth-order strain gradient model, with nonlocal and surface energy effects on the mechanical responses of the nanowire-substrate system, while the second simulation examines the sensitivity of system stiffness on nanowire and substrate properties. Finally, the last section gives the conclusions from this study. All symbolic calculations in this work were performed with the computer software Mathematica [54].

\section{NONLOCAL STRAIN GRADIENT MODEL}

The nonlocal strain gradient model $[25,29]$ was originally derived from the nonlocal elasticity theory [14], which assumed the stress at a reference point $\mathbf{x}$ is a function of the strain field at every point in the body. The original constitutive relation of the nonlocal elasticity theory was represented by the weighted averages of the contribution of the strain tensor at all points in the body to the stress tensor at the given point. This leads to the mathematical problems to get the solution for nanostructure continuum models. To overcome this limitation, Eringen [14] presented the kernel function in the form of a modified Bessel function for applying in the differential constitutive equation. However, the integral constitutive relation as suggested by Eringen [14] can be converted exactly into a corresponding differential form for some kernels [25, 29]. Later, Peddieson et al. [25] presented an alternative way to avoid the mathematical problem in the nonlocal elasticity theory by using the nonlocal strain gradient model. The constitutive relation of the nonlocal strain gradient model comes from the integral constitutive relation of the nonlocal elasticity theory [14] when the material-length scale parameter is very less than $1\left(e_{0} a<<1\right)$. Thus, the expansion of the elastic energy $W$ based on the following assumption for a uniaxial stress problem is obtained by only considering the first three terms as:

$$
\begin{aligned}
W\left[\varepsilon_{x x}(x), \varepsilon_{1}(x), \varepsilon_{2}(x)\right]=\underbrace{\frac{1}{2} E_{x x} \varepsilon_{x x}(x)^{2}}_{\text {Local term }} \\
\underbrace{+\frac{1}{2} E_{x x}\left(e_{0} a\right)^{2}\left(\varepsilon_{1}(x)\right)^{2}+\frac{1}{2} E_{x x}\left(e_{0} a\right)^{4}\left(\varepsilon_{2}(x)\right)^{2}}_{\text {Nonlocal terms }}
\end{aligned}
$$


where $E_{x x}$ is the Young modulus; $e_{0} a$ is the nonlocal-scale parameter; $\varepsilon_{x x}(x)$ is the axial strain; $\varepsilon_{1}(x)$ and $\varepsilon_{2}(x)$ are the axial strain gradients corresponding to the second and the third terms in the nonlocal strain gradient model, respectively. It needs to be emphasized that the first term on the right-hand side of Eq. (1) represents the local (conventional) strain energy (as in large-scale structural analysis), while the second and third terms represent the nonlocal strain energy (small-size-scale effect) associated with the second and the third terms of the nonlocal strain gradient model, respectively.

The elastic energy time rate of the energy functional $W$ of Eq. (1) is:

$$
\dot{W}\left[\varepsilon_{x x}(x), \varepsilon_{1}(x), \varepsilon_{2}(x)\right]=\frac{\partial W}{\partial \varepsilon_{x x}} \dot{\varepsilon}_{x x}+\frac{\partial W}{\partial \varepsilon_{1}} \dot{\varepsilon}_{1}+\frac{\partial W}{\partial \varepsilon_{2}} \dot{\varepsilon}_{2}=\sigma_{0} \dot{\varepsilon}_{x x}+\sigma_{1} \dot{\varepsilon}_{1}+\sigma_{2} \dot{\varepsilon}_{2}
$$

where a dot on top of a symbol (') denotes the time derivative; $\sigma_{0}$ is the local (conventional) normal stress; $\sigma_{1}$ and $\sigma_{2}$ are higher-order normal stresses associated with the second and the third terms of strain gradient, respectively. These normal stresses can be defined as:

$$
\begin{gathered}
\sigma_{0}=\frac{\partial W}{\partial \varepsilon_{x x}}=E_{x x} \varepsilon_{x x} \\
\sigma_{1}=\frac{\partial W}{\partial \varepsilon_{1}}=\left(e_{0} a\right)^{2} E_{x x} \varepsilon_{1} \\
\sigma_{2}=\frac{\partial W}{\partial \varepsilon_{2}}=\left(e_{0} a\right)^{4} E_{x x} \varepsilon_{2}
\end{gathered}
$$

It is clear from Eqs. (3) to (5) that the local normal stress $\sigma_{0}$ is the conjugate-work pair of the axial strain $\varepsilon_{x x}$, as shown in Eq. (3), while the higher-order normal stresses $\sigma_{1}$ and $\sigma_{2}$ are the conjugate-work pairs of the axial strain gradients $\varepsilon_{1}$ and $\varepsilon_{2}$ as demonstrated in Eqs. (4) and (5), respectively.

In order to fulfill the thermodynamics, the elastic energy time rate of the energy functional $W$ of Eq. (2) must be satisfied as following condition [33]:

$$
\int_{L}\left(\int_{A} \sigma_{x x} \dot{\varepsilon}_{x x} d A\right) d x-\int_{L}\left(\int_{A} \dot{W} d A\right) d x
$$

where $\sigma_{x x}$ is the nonlocal normal stress. By substituting Eqs. (3) to (5) into Eq. (6), the relation is:

$$
\int_{L}\left(\int_{A} \sigma_{x x} d A\right) \dot{\varepsilon}_{x x} d x-\underbrace{\int_{L}\left(\int_{A} \sigma_{0} d A\right) \dot{\varepsilon}_{x x} d x}_{\text {Local term }}-\underbrace{\int\left(\int_{\mathrm{A}} \sigma_{1} \mathrm{dA}\right) \dot{\varepsilon}_{1} \mathrm{dx}}_{\text {Second-order strain gradient term }}-\underbrace{\int_{L}\left(\int_{A} \sigma_{2} d A\right) \dot{\varepsilon}_{2} d x}_{\text {Fourth-order strain gradient term }}=0 \text { (7) }
$$


To simplify the variational form in Eq. (7) for analysis of a uniaxial problem, it can apply terms in the axial force $N_{x x}(x)$ :

$$
\int_{L} N_{x x}(x) \dot{\varepsilon}_{x x} d x-\underbrace{\int_{L} N_{0}(x) \dot{\varepsilon}_{x x} d x}_{\text {Local term }}-\underbrace{\int_{L} N_{1}(x) \dot{\varepsilon}_{1} d x}_{\text {Second-order strain gradient term }}-\underbrace{\int_{L} N_{2}(x) \dot{\varepsilon}_{2} d x}_{\text {Fourth-order strain gradient term }}=0
$$

where $N_{0}(x)=\int_{A} \sigma_{0} d A$ are the local axial forces; $N_{1}(x)=\int_{A} \sigma_{1} d A$ and $N_{2}(x)=\int_{A} \sigma_{2} d A$ are the higher-order axial forces associated with the second and the third terms of the nonlocal strain gradient model within the thermodynamic approach, respectively.

\section{SURFACE ELASTICITY THEORY}

The size-dependent effect or the so-called "surface energy" effect stems from different energy density at the surfaces of the bulk material, becoming significant when the surfaceto-volume ratio is large. This is found in various experiments and in atomistic simulations of micro/nano-scale problems $[38,41]$. To account for the effect of surfaces and interfaces, Gurtin and Murdoch [42-43] introduced a non-classical continuum model of surface elasticity under the hypothesis of a zero-thickness surface layer and perfect bonding between a solid core and an outer surface shell, as shown in Fig 1. For the analysis of the uniaxial responses in this study, the relation between the surface stress $\tau_{x x}$ and surface strain $\varepsilon_{s u r}$ (constitutive relation of surface elasticity) along the longitudinal direction are given by Gurtin and Murdoch [42-43] as:

$$
\tau_{x x}-\tau_{0}=E_{s u r} \varepsilon_{\text {sur }}
$$

where $E_{\text {sur }}$ and $\tau_{0}$ are, respectively, the surface elastic modulus and residual surface stress when the values of both of these are obtained from experiments or from atomistic simulations [38].

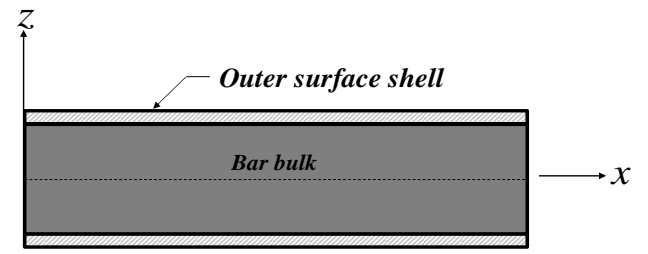

Nanowire

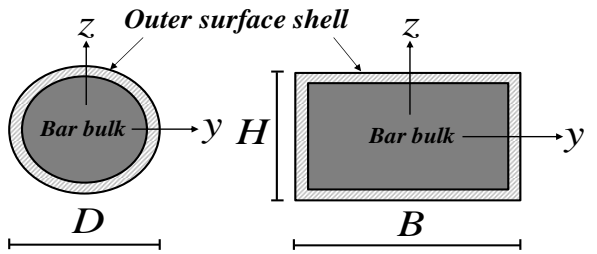

Circular section $\quad \underline{\text { Rectangular section }}$

Fig. 1 Nanowire embedded in substrate with a circular or a rectangular wire cross-section [37] 


\section{FORMULATION}

\subsection{Compatibility Equations}

Based on the small strain assumption, the sectional axial strain of the nanowire $\varepsilon_{x x}(x)$, and the sectional axial strain gradients $\varepsilon_{1}(x)$ and $\varepsilon_{2}(x)$ are related to the axial displacement of the nanowire $u_{x}(x)$ through the compatibility conditions:

$$
\begin{gathered}
\varepsilon_{x x}(x)=\frac{\partial u_{x}(x)}{\partial x} \\
\varepsilon_{1}(x)=\frac{\partial \varepsilon_{x x}(x)}{\partial x}=\frac{\partial^{2} u_{x}(x)}{\partial x^{2}} \\
\varepsilon_{2}(x)=\frac{\partial^{2} \varepsilon_{x x}(x)}{\partial x^{2}}=\frac{\partial^{3} u_{x}(x)}{\partial x^{3}}
\end{gathered}
$$

Under the hypothesis of perfect bonding between the bulk of nanowire and the surface surrounding outer surface shell [42-43], the surface displacement $u_{\text {sur }}(x)$ is perfectly compatible with the axial displacement of the nanowire $\left(u_{s u r}(x)=u_{x}(x)\right)$. Therefore, the compatibility relation of the surface surroundings involving surface strain $\varepsilon_{s u r}(x)$, surface displacement $u_{\text {sur }}(x)$, and axial displacement of the nanowire $u_{x}(x)$ is as follows:

$$
\varepsilon_{\text {sur }}(x)=\frac{\partial u_{\text {sur }}(x)}{\partial x}=\frac{\partial u_{x}(x)}{\partial x}
$$

Like the assumption regarding surface surroundings, the interaction between nanowire and substrate is assumed to be perfect based on the kinematic assumption of the Winkler foundation model [55]. Thus, the compatibility relation between the substrate deformation $\Delta_{s u b}(x)$ and the nanowire axial displacement $u_{x}(x)$ is:

$$
\Delta_{\text {sub }}(x)=u_{x}(x)
$$

\subsection{Differential Equation and Its Boundary Conditions: The Principle of Virtual Displacement}

In the displacement-based formulation, the principle of virtual displacement is employed to establish the governing differential equilibrium equation and its boundary conditions for the nanowire element embedded in the substrate, as shown in Fig. 2. The features, such as the nanowire-substrate interaction, the nonlocality, and the surface energy effect, are incorporated to the model in this procedure. 


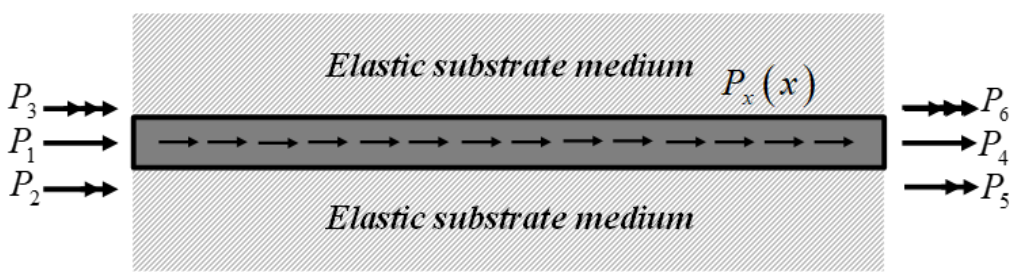

Fig. 2 Ideal nanobar element embedded in substrate

A general form of the total virtual work expression is:

$$
\delta W=\delta W_{\text {int }}+\delta W_{\text {ext }}=0
$$

where $\delta W$ is the total virtual work of the bar-substrate element system; $\delta W_{\text {int }}$ is the internal virtual work; and $\delta W_{\text {ext }}$ is the external virtual work.

The internal virtual work $\delta W_{\text {int }}$ and the external virtual work $\delta W_{\text {ext }}$ of Eq. (15) can be expressed as:

$$
\begin{aligned}
& \delta W_{\text {int }}=\underbrace{\int_{L}\left(\int_{A} \sigma_{x x}(x) d A\right) \delta \varepsilon_{x x}(x) d x}_{\text {Nanowire term }}+\underbrace{\int_{L}\left(\oint_{\Gamma}\left(\tau_{x x}(x)-\tau_{0}\right) d \Gamma\right) \delta \varepsilon_{\text {sur }}(x) d x}_{\text {Surface energy term }} \\
& +\underbrace{\int_{L} D_{\text {sub }}(x) \delta \Delta_{\text {sub }}(x) d x}_{\text {Substrate medium term }}
\end{aligned}
$$

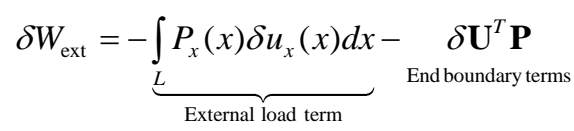

where $D_{s u b}(x)$ is the substrate interactive force and the conjugate-work pair of the substrate deformation $\Delta_{s u b}(x) ; P_{x}(x)$ is the axial distributed load; $\mathbf{U}=\left\{\begin{array}{lllllll}U_{1} & U_{2} & U_{3} & U_{4} & U_{5} & U_{6}\end{array}\right\}^{T}$ is the displacement vector that contains the end displacements of the nanowire element; and $\mathbf{P}=\left\{\begin{array}{llllll}P_{1} & P_{2} & P_{3} & P_{4} & P_{5} & P_{6}\end{array}\right\}^{T}$ is the force vector that contains the end forces of the nanowire element.

Considering the variational form of Eqs. (7) and (8), the internal virtual works in Eq. (16) becomes:

$$
\begin{aligned}
\delta W_{\text {int }} & =\overbrace{\int_{\int_{0}}^{N_{N}(x) \delta \varepsilon_{x x}(x) d x}}^{\text {Local term }}+\overbrace{\int_{L} N_{1}(x) \delta \varepsilon_{1}(x) d x}^{\text {Second-order strain gradient term }}+\overbrace{\int_{L} N_{2}(x) \delta \varepsilon_{2}(x) d x}^{\text {Forth-order strain gradient term }} \\
& +\underbrace{\int_{L} N_{\text {sur }}(x) \delta \varepsilon_{\text {sur }}(x) d x}_{\text {Sunowire terms }}+\underbrace{\int_{L} D_{\text {sub }}(x) \delta \Delta_{\text {sub }}(x) d x}_{\text {Substrate medium term }}
\end{aligned}
$$

where $N_{\text {sur }}(x)=\oint_{\Gamma}\left(\tau_{x x}(x)-\tau_{0}\right) d \Gamma$ represents the equivalent axial force from the surface stresses. By substituting the compatibility equations of Eqs. (10) to (14) into the internal 
virtual work $\delta W_{\text {int }}$ of Eq. (18) and then combining with the external virtual work $\delta W_{\text {ext }}$ of Eq. (17), the total virtual work of nanowire-substrate element system of Eq. (15) is:

$$
\begin{aligned}
\delta W & =\int_{L} N_{0}(x) \frac{\partial \delta u_{x}(x)}{\partial x} d x+\int_{L} N_{1}(x) \frac{\partial^{2} \delta u_{x}(x)}{\partial x^{2}} d x+\int_{L} N_{2}(x) \frac{\partial^{3} \delta u_{x}(x)}{\partial x^{3}} d x \\
& +\int_{L} N_{s u r}(x) \frac{\partial \delta u_{x}(x)}{\partial x} d x+\int_{L} D_{s u b}(x) \delta u_{x}(x) d x-\int_{L} P_{x}(x) \delta u_{x}(x) d x \\
& -\delta \mathbf{U}^{T} \mathbf{P}=0
\end{aligned}
$$

To move the all differential operators of the axial displacement $u_{x}(x)$ into the sectional force resultants, integration by parts is applied to Eq. (19):

$$
\begin{aligned}
\delta W & =\int_{L} \delta u_{x}(x)\left[-\frac{\partial^{3} N_{2}(x)}{\partial x^{3}}+\frac{\partial^{2} N_{1}(x)}{\partial x^{2}}-\frac{\partial N_{0}(x)}{\partial x}-\frac{\partial N_{\text {sur }}(x)}{\partial x}+D_{\text {sub }}(x)-P_{x}(x)\right] d x \\
& +\delta u_{x}(x)\left[\frac{\partial^{2} N_{2}(x)}{\partial x^{2}}-\frac{\partial N_{1}(x)}{\partial x}+N_{0}(x)+N_{\text {sur }}(x)\right]_{x=0}^{x=L} \\
& +\frac{\partial \delta u_{x}(x)}{\partial x}\left[-\frac{\partial N_{2}(x)}{\partial x}+N_{1}(x)\right]_{x=0}^{x=L}+\frac{\partial^{2} \delta u_{x}(x)}{\partial x^{2}}\left[N_{2}(x)\right]_{x=0}^{x=L} \\
& -\delta \mathbf{U}^{T} \mathbf{P}=0
\end{aligned}
$$

Following the Cartesian sign convention, Eq. (20) can be expressed as:

$$
\begin{aligned}
\delta W & =\int_{L} \delta u_{x}(x)\left[-\frac{\partial^{3} N_{2}(x)}{\partial x^{3}}+\frac{\partial^{2} N_{1}(x)}{\partial x^{2}}-\frac{\partial N_{0}(x)}{\partial x}-\frac{\partial N_{s u r}(x)}{\partial x}+D_{s u b}(x)-P_{x}(x)\right] d x \\
& -\delta U_{1}\left[\left(\frac{\partial^{2} N_{2}(x)}{\partial x^{2}}-\frac{\partial N_{1}(x)}{\partial x}+N_{0}(x)+N_{s u r}(x)\right)_{x=0}+P_{1}\right] \\
& -\delta U_{2}\left[\left(-\frac{\partial N_{2}(x)}{\partial x}+N_{1}(x)\right)_{x=0}+P_{2}\right]-\delta U_{3}\left[\left(N_{2}(x)\right)_{x=0}+P_{3}\right] \\
& +\delta U_{4}\left[\left(\frac{\partial^{2} N_{2}(x)}{\partial x^{2}}-\frac{\partial N_{1}(x)}{\partial x}+N_{0}(x)+N_{s u r}(x)\right)_{x=L}-P_{4}\right] \\
& +\delta U_{5}\left[\left(-\frac{\partial N_{2}(x)}{\partial x}+N_{1}(x)\right)_{x=L}-P_{5}\right]+\delta U_{6}\left[\left(N_{2}(x)\right)_{x=L}-P_{6}\right] \\
& -\delta \mathbf{U}^{T} \mathbf{P}=0
\end{aligned}
$$

Considering the arbitrary variable of $\delta u_{x}(x)$ in Eq. (21), the governing differential equilibrium equation for the proposed nanowire element embedded in elastic substrate is:

$$
-\frac{\partial^{3} N_{2}(x)}{\partial x^{3}}+\frac{\partial^{2} N_{1}(x)}{\partial x^{2}}-\frac{\partial N_{0}(x)}{\partial x}-\frac{\partial N_{s u r}(x)}{\partial x}+D_{s u b}(x)-P_{x}(x)=0
$$


From Eq. (21), the boundary force conditions at the ends of an element are also obtained by considering arbitrary $\delta \mathbf{U}$ :

$$
\begin{aligned}
& P_{1}=-\left[\frac{\partial^{2} N_{2}(x)}{\partial x^{2}}-\frac{\partial N_{1}(x)}{\partial x}+N_{0}(x)+N_{s u r}(x)\right]_{x=0} ; \\
& P_{2}=-\left[-\frac{\partial N_{2}(x)}{\partial x}+N_{1}(x)\right]_{x=0} ; P_{3}=-\left[N_{2}(x)\right]_{x=0} ; \\
& P_{4}=\left[\frac{\partial^{2} N_{2}(x)}{\partial x^{2}}-\frac{\partial N_{1}(x)}{\partial x}+N_{0}(x)+N_{\text {sur }}(x)\right]_{x=L} ; \\
& P_{5}=\left[-\frac{\partial N_{2}(x)}{\partial x}+N_{1}(x)\right]_{x=L} ; P_{6}=\left[N_{2}(x)\right]_{x=L}
\end{aligned}
$$

\subsection{Force-Deformation Relations}

The force-deformation relations of the axial resultants for nanowire are established by inserting the compatibility equations of Eqs. (10) to (12) into the relations in Eqs. (3) to (5) and considering stress in term of axial force, resulting in the following relations:

$$
\begin{gathered}
N_{0}(x)=E_{x x} A \frac{\partial u_{x}(x)}{\partial x} \\
N_{1}(x)=E_{x x} A\left(e_{0} a\right)^{2} \frac{\partial^{2} u_{x}(x)}{\partial x^{2}} \\
N_{2}(x)=E_{x x} A\left(e_{0} a\right)^{4} \frac{\partial^{3} u_{x}(x)}{\partial x^{3}}
\end{gathered}
$$

where $A=\int_{A} d A$ represents the cross-section area. The force-deformation relation of the axial force induced by the surface stress can be found by substituting the compatibility condition Eq. (13) into Eq. (9), and considering this in terms of the axial resultant. Thus, the force-deformation relation of surface surroundings is

$$
N_{s u r}(x)=E_{s u r} \Gamma \frac{\partial u_{x}(x)}{\partial x}
$$

where $\Gamma=\oint_{\Gamma} d \Gamma$ is the section perimeter of nanowire. Similarly, the relation between substrate interactive force $D_{\text {sub }}(x)$ and the substrate deformation $\Delta_{s u b}(x)$ can be expressed by enforcing the compatibility relation of Eq. (14) as:

$$
D_{\text {sub }}(x)=k_{s} \Delta_{\text {sub }}(x)=k_{s} u_{x}(x)
$$

where $k_{s}$ represents the elastic modulus of the substrate medium. The compatibility relations, equilibrium equation, and force-deformation relations of the proposed nanowire element 
embedded in elastic substrate media as demonstrated above, can be conveniently summarized for the whole model formulation in a Tonti's diagram [56], as shown Fig. 3.

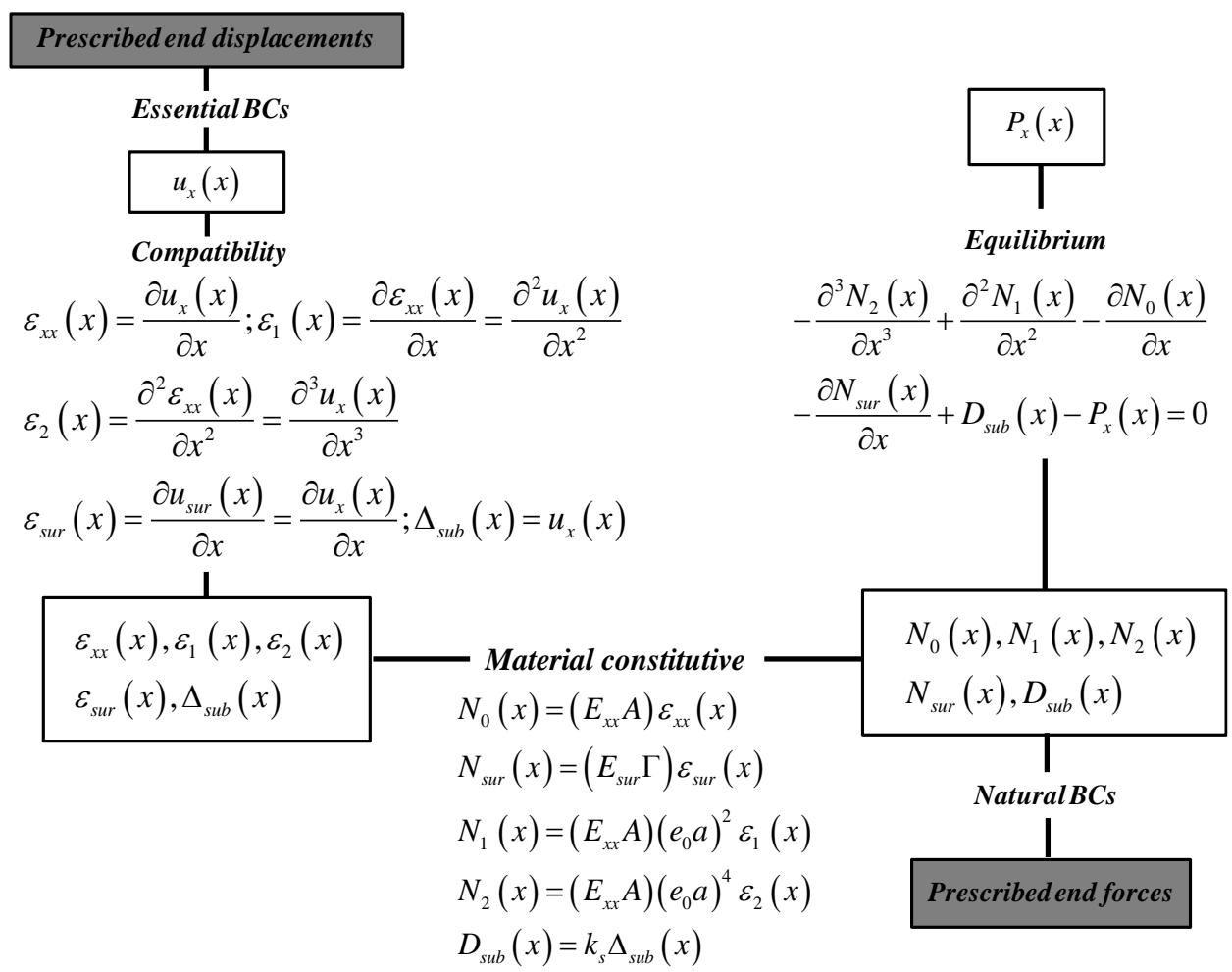

Fig. 3 Tonti's diagram for the fourth-order nonlocal strain gradient model of nanowire embedded in elastic substrate

\section{4. "Exact”' Displacement Shape Functions}

According to the force-deformation relations in Eqs. (24) to (28), the governing differential equilibrium equation in Eq. (22) can be rewritten in terms of the axial displacement $u_{x}(x)$ as:

$$
-E_{x x} A\left(e_{0} a\right)^{4} \frac{\partial^{6} u_{x}(x)}{\partial x^{6}}+E_{x x} A\left(e_{0} a\right)^{2} \frac{\partial^{4} u_{x}(x)}{\partial x^{4}}-\left(E_{x x} A\right)_{e f f} \frac{\partial^{2} u_{x}(x)}{\partial x^{2}}+k_{s} u_{x}(x)=P_{x}(x)
$$

where $\left(E_{x x} A\right)_{\text {eff }}=E_{x x} A+E_{\text {sur }} \Gamma$ represents the effective nanowire stiffness accounting for the contribution of the solid core and the outer surface. The effective nanowire stiffnesses $\left(E_{x x} A\right)_{e f f}$ for circular and rectangular sections are given by Juntarasaid et al. [57] as:

$$
\begin{array}{ll}
\left(E_{x x} A\right)_{e f f}=E_{x x} \frac{\pi}{4} D^{2}+E_{\text {sur }} \pi D & \text { for circular section } \\
\left(E_{x x} A\right)_{e f f}=E_{x x} B H+2 E_{\text {sur }}(B+H) & \text { for rectangular section }
\end{array}
$$


where $D$ is the diameter of circular nanowire cross-section; and $B$ and $H$ are, respectively, the width and height of a rectangular section.

It needs to be pointed out that the sixth-order governing differential equilibrium equation in Eq. (29) represents the state equilibrium for the fourth-order strain gradient model of the nanowire embedded in elastic substrate, with inclusion of the surface energy effect. When the third term of the nonlocal strain gradient model in Eq. (22) vanishes $\left(N_{2}(x)=0\right)$, the governing differential equilibrium equation in Eq. (29) will be reduced to a second-order nonlocal strain gradient model proposed by Sea-Long et al. [37]. Furthermore, the governing differential equilibrium equation in Eq. (29) can be transformed into the local bar-elastic medium system when the nonlocal-scale parameter and the surface energy effect are ignored $\left(e_{0} a=E_{s u r}=0\right)$. The general form of differential equation in Eq. (29) is found in several engineering applications. For example, in a beam on elastic threeparameter foundation as reported by Morfidis [52] and by Avramidis and Morfidis [53] etc.

The natural boundary conditions (end boundary force terms) in terms of axial displacement $u_{x}(x)$ can be expressed by the force-deformation relations in Eqs. (24) to (27) in Eq. (23), giving

$$
\begin{aligned}
& P_{1}=-[\underbrace{E_{x x} A\left(e_{0} a\right)^{4} \frac{\partial^{5} u_{x}(x)}{\partial x^{5}}}_{\text {Fourth-order strain gradient term }}-\underbrace{E_{x x} A\left(e_{0} a\right)^{2} \frac{\partial^{3} u_{x}(x)}{\partial x^{3}}}_{\text {Second-order strain gradient term }}+\underbrace{\left(E_{x x} A\right)_{\text {eff }} \frac{\partial u_{x}(x)}{\partial x}}_{\text {Local term }}]_{x=0} ; \\
& P_{2}=-[\underbrace{-E_{x x} A\left(e_{0} a\right)^{4} \frac{\partial^{4} u_{x}(x)}{\partial x^{4}}}_{\text {Fourth-order strain gradient term }}+\underbrace{E_{x x} A\left(e_{0} a\right)^{2} \frac{\partial^{2} u_{x}(x)}{\partial x^{2}}}_{\text {Second-order strain gradient term }}]_{x=0} ; P_{3}=-[\underbrace{E_{x x} A\left(e_{0} a\right)^{4} \frac{\partial^{3} u_{x}(x)}{\partial x^{3}}}_{\text {Fourth-order strain gradient term }}]_{x=0} ; \\
& P_{4}=[\underbrace{E_{x x} A\left(e_{0} a\right)^{4} \frac{\partial^{5} u_{x}(x)}{\partial x^{5}}}_{\text {Fourth-order strain gradient term }}-\underbrace{E_{x x} A\left(e_{0} a\right)^{2} \frac{\partial^{3} u_{x}(x)}{\partial x^{3}}}_{\text {Second-order strain gradient term }}+\underbrace{\left(E_{x x} A\right)_{e f f} \frac{\partial u_{x}(x)}{\partial x}}_{\text {Local term }}]_{x=L} ; \\
& P_{5}=[\underbrace{-E_{x x} A\left(e_{0} a\right)^{4} \frac{\partial^{4} u_{x}(x)}{\partial x^{4}}}_{\text {Fourth-order strain gradient term }}+\underbrace{E_{x x} A\left(e_{0} a\right)^{2} \frac{\partial^{2} u_{x}(x)}{\partial x^{2}}}_{\text {Second-order strain gradient term }}]_{x=L} ; P_{6}=[\underbrace{E_{x x} A\left(e_{0} a\right)^{4} \frac{\partial^{3} u_{x}(x)}{\partial x^{3}}}_{\text {Fourth-order strain gradient term }}]_{x=L}
\end{aligned}
$$

It can be observed from the boundary conditions of Eq. (31) that the end boundary forces associated to the local system, $P_{1}$ and $P_{4}$, are not resisted only by the local axial force but also by the higher-order axial forces from the gradient terms. Furthermore, the statically equivalent axial force due to the higher-order strain gradient terms $N_{1}(0), N_{2}(0)$, $N_{1}(L)$, and $N_{2}(L)$ must satisfy kinematics of the nanowire element at the end boundaries, thus resulting zeroes for $-\left(\partial N_{2}(0) / \partial x\right)+N_{1}(0), N_{2}(0),-\left(\partial N_{2}(L) / \partial x\right)+N_{1}(L)$, and $N_{2}(L)$.

In the current work, the analytical solutions of the sixth-order governing differential equilibrium equation in Eq. (29) are adapted and applied from the general solutions of the beam problem on the Kerr-type foundation, proposed by Morfidis [52] and Avramidis and Morfidis [53]. This leads to the so-called "exact" displacement shape functions:

$$
u_{x}(x)=\psi_{1}(x) C_{1}+\psi_{2}(x) C_{2}+\psi_{3}(x) C_{3}+\psi_{4}(x) C_{4}+\psi_{5}(x) C_{5}+\psi_{6}(x) C_{6}
$$


where $\psi_{1}(x)$ to $\psi_{6}(x)$ are the functions associated with solutions of the sixth-order differential equation; and $C_{1}$ to $C_{6}$ are the generalized coordinates corresponding to the geometric boundary conditions. More details of the exact displacement shape functions are given in Appendix A.

\section{NUMERICAL SIMULATIONS}

In this section, two numerical simulations are demonstrated and assessed for the mechanical characteristics of nanowire embedded in an elastic substrate. The first simulation investigates the mechanical responses of that nanowire to illustrate the influences of higher-order terms in the strain gradient, nonlocal, and surface energy effects on the nanowire responses. The second simulation examines the influences of system parameters on the effective Young's modulus of the nanowire system, and the element stiffness at the end of the nanowire model.

\subsection{Simulation I: Nonlocal Strain Gradient Models vs Local Model in the Analysis of a Nanowire embedded in an Elastic Substrate}

A free-free silver nanowire embedded in the elastic substrate under the tensile force $P_{E n d}$ of $100,000 \mathrm{nN}$ at the right-hand side of the member end, as shown in Fig. 4, is studied in this simulation.

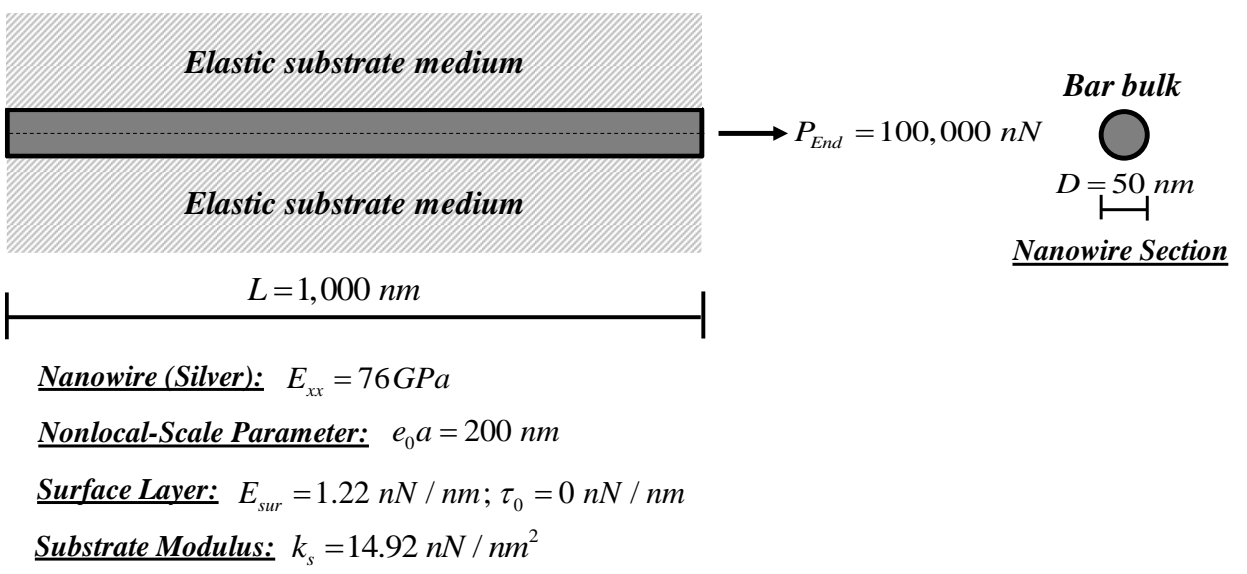

Fig. 4 Simulation I: System with a free-free nanowire embedded in an elastic substrate for response analysis

The material and geometric properties of the nanowire come from the publication of Juntarasaid et al. [57], with $76 \mathrm{GPa}$ bulk modulus of the silver nanowire $E_{x x}$; and $50 \mathrm{~nm}$ diameter $D$. The length of the nanowire $L$ is assumed to be $1,000 \mathrm{~nm}$. The nonlocal-scale parameter $e_{0} a$ is set at $200 \mathrm{~nm}$. This value has been found in several studies on characteristics of nanostructures [37, 49-50]. The surface energy choice follows the publication of He and Lilley [58] with elastic surface modulus $E_{\text {sur }}=1.22 \mathrm{nN} / \mathrm{nm}$, while the residual surface stress 
is assumed zero ( $\tau_{0}=0 \mathrm{nN} / \mathrm{nm}$ ). These values correspond to the crystallographic direction [001] as introduced by Shenoy [59]. The characteristics of a polymer as the surrounding medium are represented by the elastic substrate modulus $k_{\mathrm{s}}=14.92 \mathrm{nN} / \mathrm{nm}^{2}$, given by Liew et al. [60].

In order to assess how nonlocal-scale effect, surface energy, and the higher-order terms of the strain gradient affect the nanowire's responses, three analytical models of such responses are compared. These are the proposed model; the second-order nonlocal strain gradient nanowire-substrate model of Sae-Long et al. [37]; and the local bar model embedded in elastic substrate. It needs to be noted that the so-called "local" model does not consider the size-scale effect. Furthermore, each analytical model gives two systems for the discussion of surface energy effect, namely the system with surface effect and the system without surface effect. Thus, there are six cases to analyze by simulations.

Fig. 5 superimposes the axial displacement along a nanowire's length obtained from the six analytical cases. This diagram indicates that the influence of the nonlocal effect on nanowire displacement is more pronounced than the surface energy effect, especially at the end of the nanowire under tensile load. The nonlocality makes the system distinctly stiffer, while the additional term in the fourth-order nonlocal strain gradient makes the element slightly stiffer, as clearly observed when compared with the local model or with the second-order strain gradient bar model. The surface energy effect slightly affected system stiffness by stiffening it when nonlocality is included. Furthermore, it can be observed that the substrate modulus is in effect nullified when nonlocality is ignored. This matches prior observations in several bar-substrate models [37, 49-50].

Similar to the observations in Fig. 5, the distribution of axial strain in Fig. 6 and the distribution of axial force in Fig. 7 are affected by the nonlocality, the higher-order terms in strain gradient, and the surface energy effects. It can see in Fig. 6 that the axial strain of the bar-substrate system distinctly differs between the local model and a set of nonlocal strain gradient models, especially at the end of the nanowire under tensile load. The axial strain from the nonlocal strain gradient models tends to spread smoothly from the end load, while the axial strain associated with the local model increases abruptly. The maximum axial strains differ between the local model and the proposed model by approximately about 3.10 times, while the maxima between the second-order model and the proposed fourth-order model differ approximately about $3.28 \%$. The axial force distribution in Fig. 7 clearly shows that the additional terms in higher-order nonlocal strain gradient models influence the axial-force action. There is a change in the axial force in compression (negative value) for both the second-order strain gradient model and the proposed fourth-order strain gradient. These results are associated with the higher-order governing differential equation of each model, seen in Eq. (29), and correspond to the static indeterminacy of the nanowire-substrate system. 


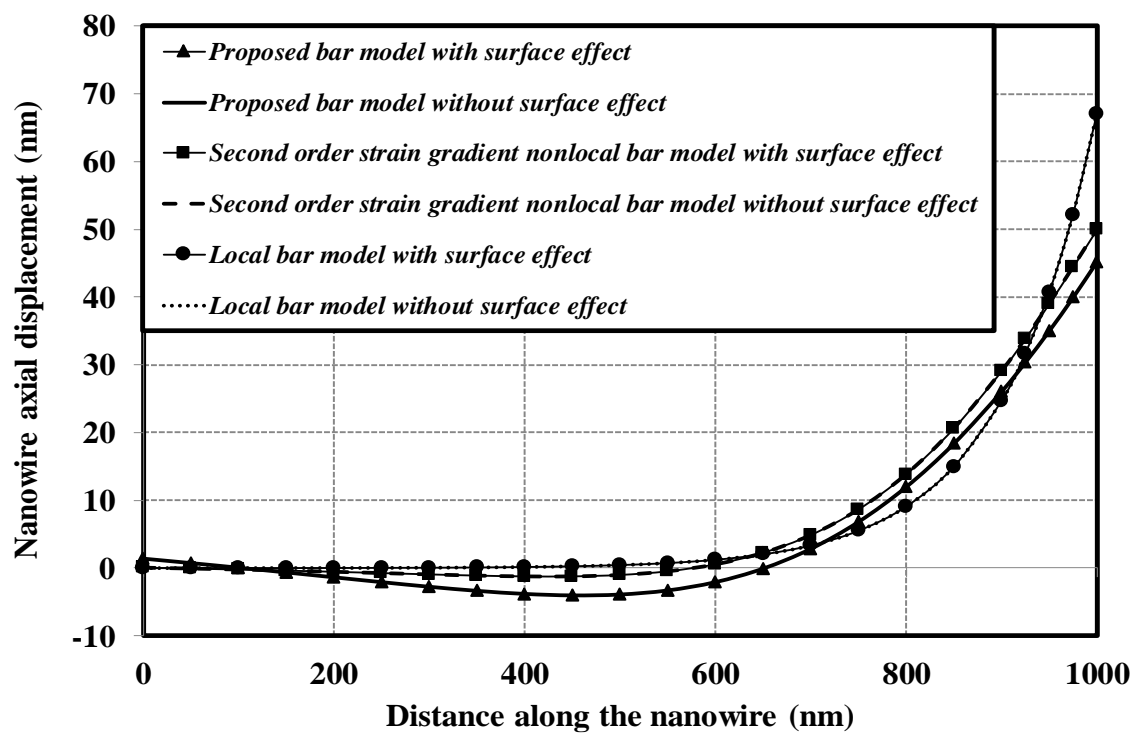

Fig. 5 Nanowire axial displacement versus distance along the nanowire

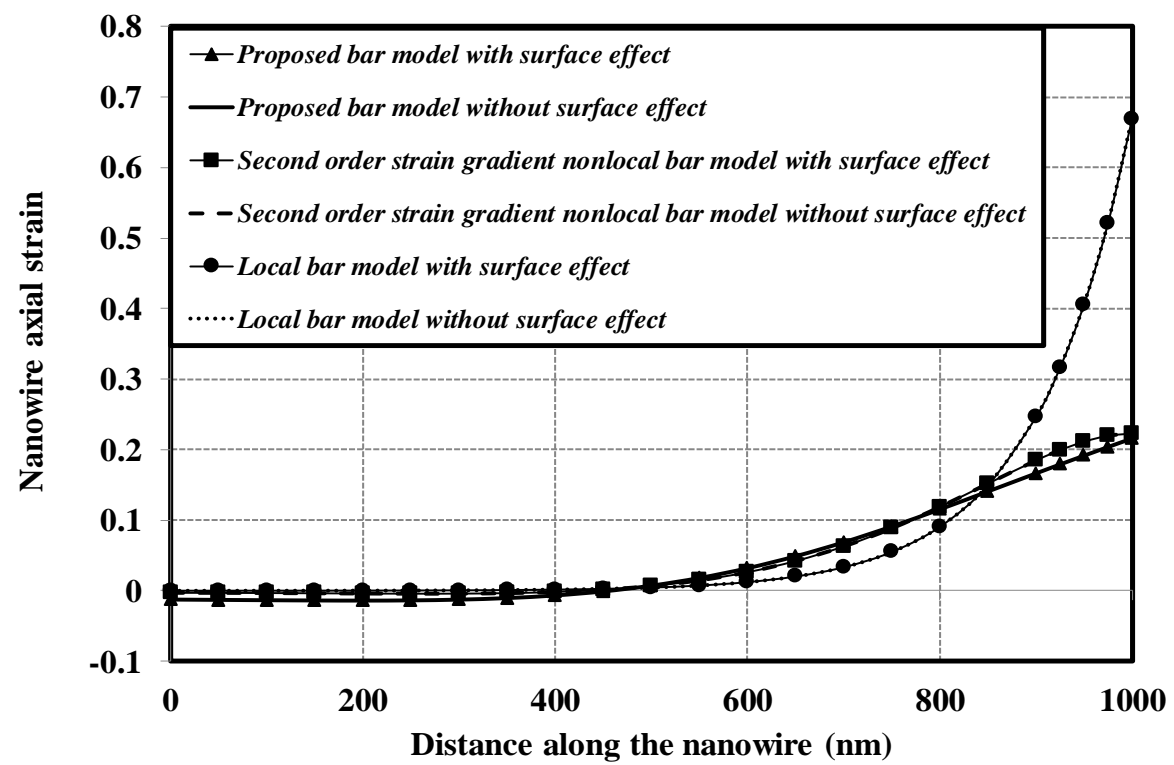

Fig. 6 Nanowire axial strain versus distance along the nanowire 


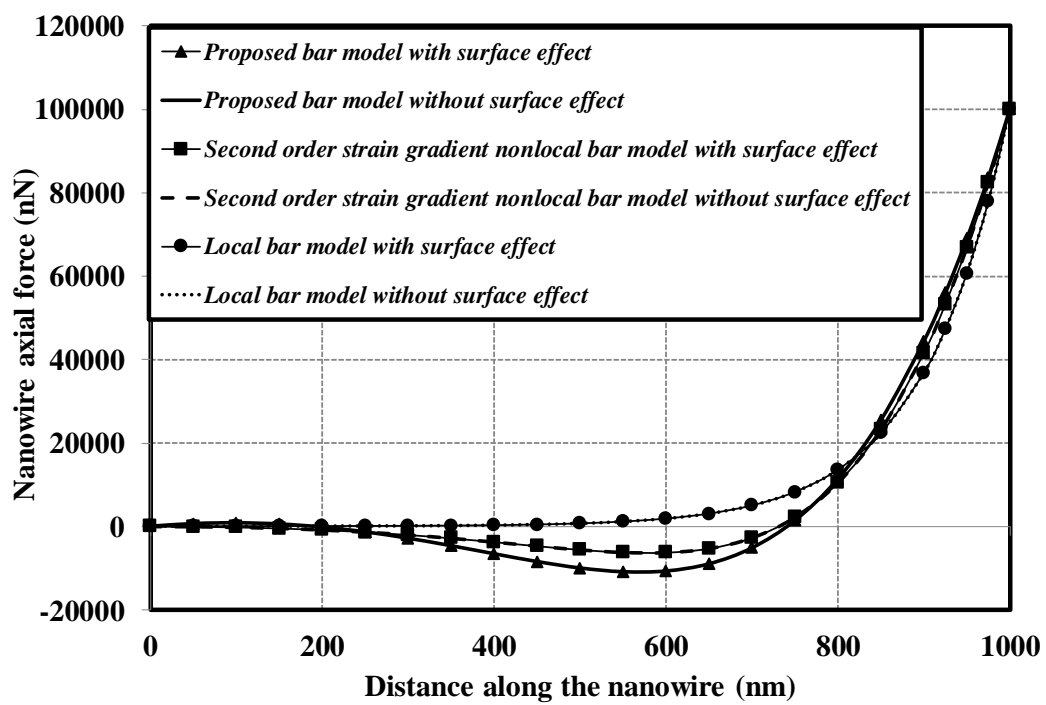

Fig. 7 Nanowire axial force versus distance along the nanowire

Fig. 8 illustrates the distribution of substrate interaction force along the nanowire for the six cases. The shapes in this diagram are similar to the axial displacement in Fig. 5. This result does not surprise because the substrate interactive force is directly obtained from the axial displacement based on the hypothesis of the Winkler-foundation model [55]. Therefore, the additional higher-order terms in the fourth-order nonlocal strain gradient model, nonlocal, and surface energy effects on this response are similar to the axial displacement in Fig. 5.

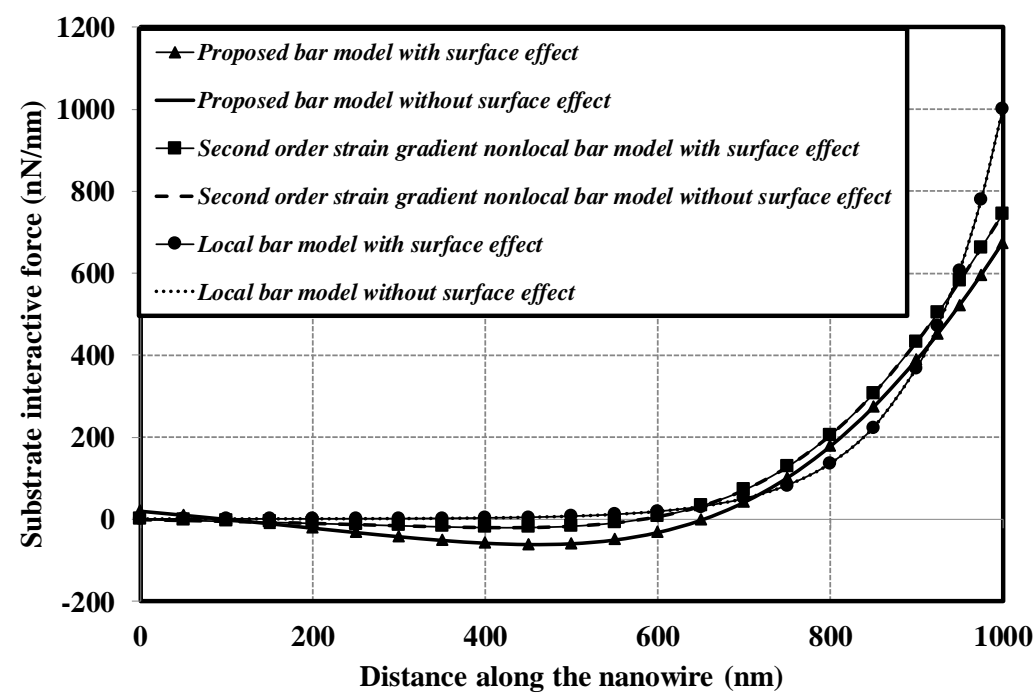

Fig. 8 Substrate interaction force versus distance along the nanowire 


\subsection{Simulation II: Parametric Study}

This simulation study investigated the sensitivity of the effective Young's modulus on nanowire and substrate parameters of the fourth-order and second-order nonlocal strain gradient models for the problem of a nanowire embedded in the substrate medium. The nanowire-substrate system under the tensile force $P_{E n d}$ of $10 \mathrm{nN}$ at the free-end right-hand side member, as shown in Fig. 9, is employed to study herein.

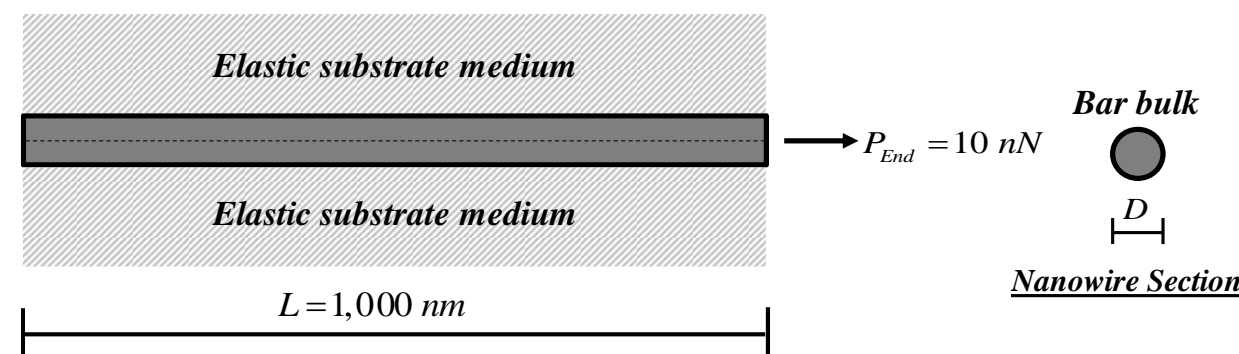

Nanowire (Anodic Alumina): $E_{x x}=70 \mathrm{GPa}$

Nonlocal-Scale Parameter: $e_{0} a=0,50,100,150,200 \mathrm{~nm}$

Surface Layer: $\quad E_{\text {sur }}=5.1882 \mathrm{nN} / \mathrm{nm} ; \tau_{0}=0 \mathrm{nN} / \mathrm{nm}$

Substrate Modulus: $k_{s}=10,25,50,100 \mathrm{nN} / \mathrm{nm}^{2}$

Nanowire Diameter: $\quad D=5,10,15,20,25,30 \mathrm{~nm}$

Fig. 9 Simulation II: System with a free-free nanowire embedded in an elastic substrate for parametric study

The material properties of nanowire and its surface energy come from the publication of Assadi et al. [61], with $70 \mathrm{GPa}$ bulk modulus of the anodic alumina nanowire $E_{x x}$; and $5.1882 \mathrm{nN} / \mathrm{nm}$ elastic surface modulus $E_{\text {sur }}$. The length of the nanowire $L$ is fixed with $1,000 \mathrm{~nm}$ while the residual surface stress $\tau_{0}$ is assumed zero. To investigate the sensitivity of the effective Young's modulus, the variation of the nanowire and substrate parameters include the nonlocal-scale parameter $e_{0} a$ with $0-200 \mathrm{~nm}$, the nanowire diameter $D$ with $5-30 \mathrm{~nm}$, and the substrate modulus $k_{\mathrm{s}}$ with $10-100 \mathrm{nN} / \mathrm{nm}^{2}$.

The method to examine the influence of the size effects on the effective Young's modulus $E_{\text {eff }}$ was adapted from He and Lilley [58] and Jiang and Yan [62], and has been widely used $[37,49]$. In the first step, the end displacement $u_{\text {End }}$ including the nonlocal and surface effects was determined from the proposed model and the second-order nonlocal strain gradient model of Sae-Long et al. [37], as shown on the left-hand side of Eq. (33). Then, the effective Young's modulus $E_{\text {eff }}$ is solved from Eq. (33).

$$
u_{\text {End }}=\frac{P_{\text {End }} \operatorname{coth}\left(\sqrt{\frac{k_{s}}{E_{e f f} A} L}\right)}{\sqrt{k_{s} E_{e f f} A}}
$$


It needs to be pointed out that the right-hand side of Eq. (33) is the end displacement based on the classical bar model on elastic foundation [63] (without nonlocal and surface effects). Therefore, the meaning of the effective Young's modulus $E_{\text {eff }}$ in Eq. (33) is the value of Young's modulus that makes the displacement in the local nanowire-substrate system equal to the displacement in the nonlocal nanowire-substrate system with the surface effect.

From the parametric study, it is clear that in all the cases shown in Fig. 10 the effective Young's modulus $E_{\text {eff }}$ increased with the nonlocal-scale parameter $e_{0} a$ and decreased with the nanowire diameter $D$, especially with larger magnitudes of the substrate modulus $k_{\mathrm{s}}$. The nanowire-substrate interaction with nonlocal and surface energy effects plays an important role in scale effects on the effective Young's modulus $E_{\text {eff. }}$ Furthermore, it can be observed that the variation of the effective Young's modulus $E_{\text {eff }}$ due to the nanowire diameter $D$ is independent on the substrate modulus $k_{\mathrm{s}}$, when the nonlocal effect is not included, as shown in Eq. (29). Finally, the added higher-order term (fourth-order nonlocal strain gradient term) of the proposed model makes the effective Young's modulus $E_{\text {eff }}$ increase when compared to the second-order nonlocal strain gradient model [37].

In order to investigate the effects of the added higher-order term in the nonlocal strain gradient model on the end stiffness of the nanowire-substrate system, this study defined the end stiffness as:

$$
K_{s t r}=\frac{P_{E n d}}{u_{E n d}} \quad \text { and } \quad K_{c}=\frac{P_{E n d}}{u_{E n d}}
$$

where $K_{s t r}$ and $K_{c}$ are the end stiffnesses corresponding to the analytical axial displacement $u_{E n d}$ as obtained from the nonlocal strain gradient models and the local model, respectively. This approach modifies the method to determine the contact stiffness from Jiang and Yan [62], Khajeansari et al. [64], and Limkatanyu et al. [49].

Fig. 11 presents the normalized end stiffness $K_{s t r} / K_{c}$ with varied nanowire diameter $D$, nonlocal-scale parameter $e_{0} a$, and substrate modulus $k_{s}$. Based on these results, the influences of small-scale, size-dependent, and nanowire-substrate interaction effects on stiffness matches the observations in the parametric study of Fig. 10. The gradient terms are nullified when nonlocality is not included $\left(e_{0} a=0\right)$. Furthermore, the influence of the higher-order term in the fourth-order nonlocal strain gradient model is more pronounced than that of the highest term in the second-order nonlocal strain gradient model, especially when the diameter of the nanowire is small and the nonlocal-scale parameter and the substrate modulus are comparatively large. This observation is clear when compared to the end boundary forces of each model, seen in Eq. (31). 

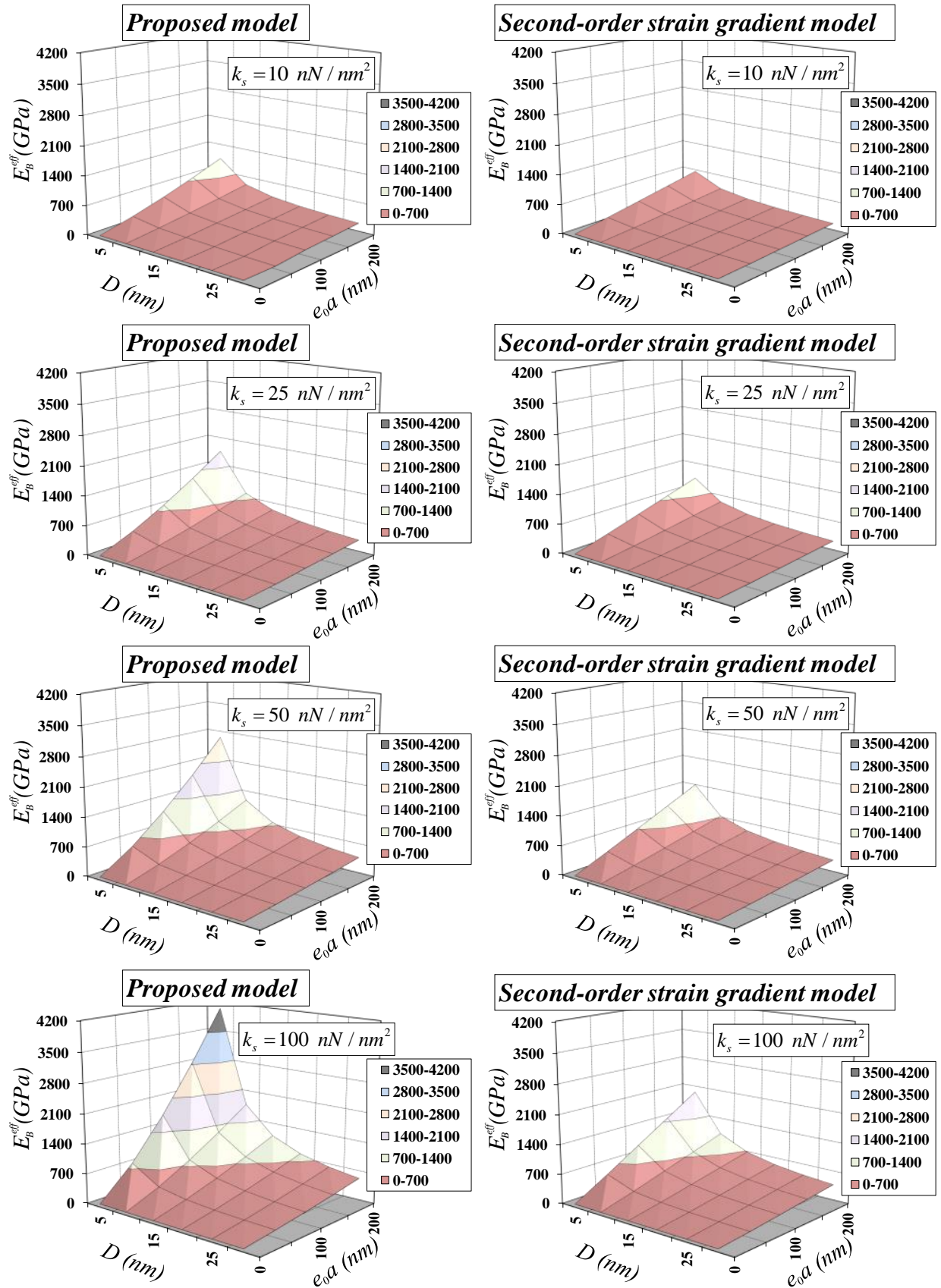

Fig. 10 Sensitivity of the effective Young's modulus to nanowire diameter, nonlocal scale parameter, and substrate modulus obtained from the proposed model and the second-order nonlocal strain gradient model 

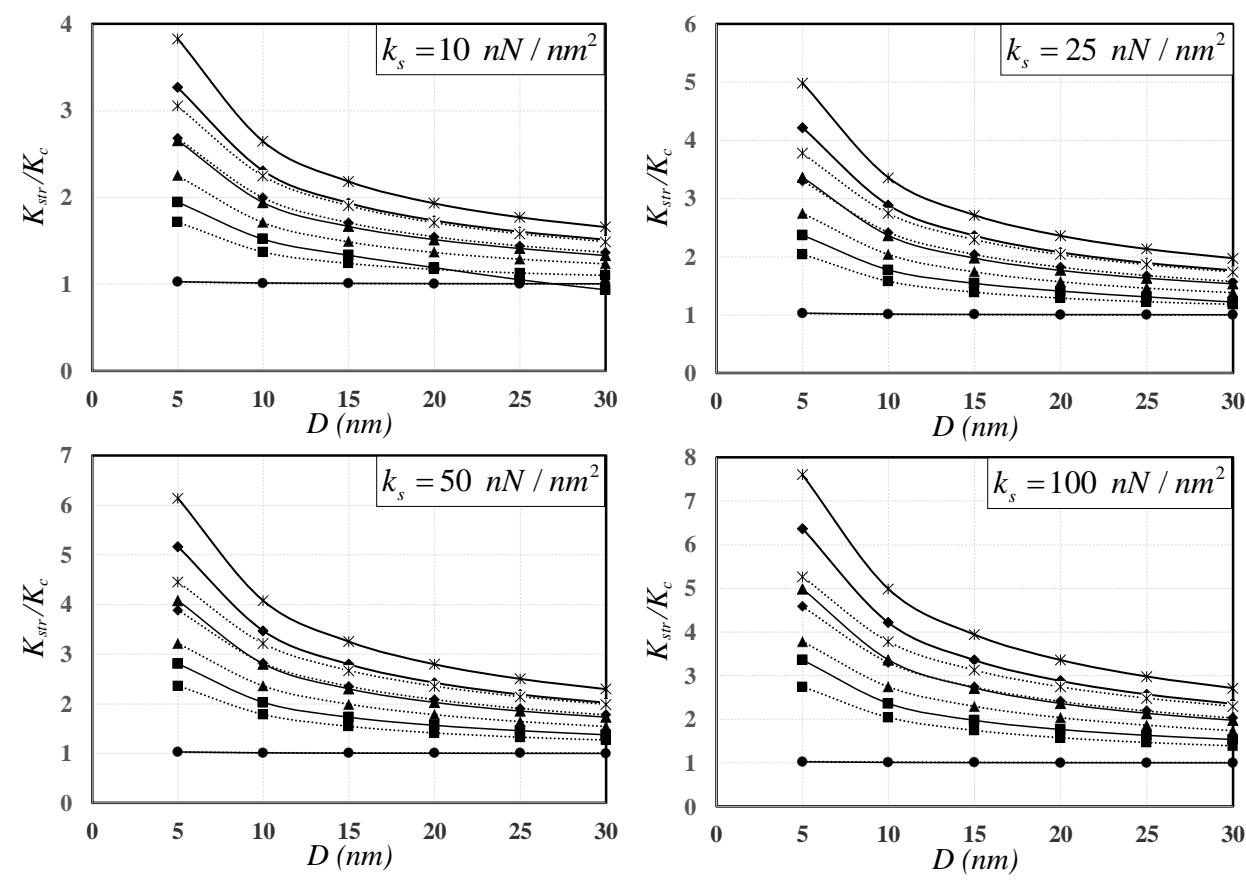

$$
\begin{aligned}
& \text { - } e_{0} a=0 \mathrm{~nm} \quad \Delta e_{0} a=100 \mathrm{~nm} \quad * \quad e_{0} a=200 \mathrm{~nm} \\
& \text { - } e_{0} a=50 \mathrm{~nm} \quad e_{0} a=150 \mathrm{~nm}
\end{aligned}
$$

Fig. 11 Variation of normalized end stiffness with diameter of nanowire for various nonlocal scale parameters and various substrate moduli

\section{CONCLUSIONS}

This paper presented a new bar-substrate model considering nonlocal and surface energy effects in the analysis of a nanowire embedded in an elastic substrate. The features of the proposed bar model include nanostructure-substrate interaction, size-scale (nonlocal) effect and size-dependent (surface) effect. The nonlocal and surface energy effects are, respectively, represented through a fourth-order nonlocal strain gradient model and the Gurtin-Murdoch continuum model, while the interaction between bar and substrate is modeled based on the Winkler foundation model. The proposed model is in a displacementbased formulation, so the governing differential equilibrium equation and its boundary conditions were determined from the principle of virtual displacement. Analytical solutions were directly obtained by solving the sixth-order governing differential equation, and were employed to provide analytical responses of the nanowire system in this study. The performance of the proposed model in characterizing the nanowire-substrate system was assessed from two numerical simulations. 
The first simulation demonstrates that nonlocality, added higher-order terms in the strain gradient theory, and surface energy effects stiffen the nanowire-substrate system. This matches the second parametric simulation study. Furthermore, both simulations indicate that the influence of nonlocality on system responses is stronger than of the surface energy effect, and the substrate modulus is nullified when nonlocality is ignored. Finally, the influence of the added term in the fourth-order nonlocal strain gradient model increased with the nonlocal-scale parameter $e_{0} a$ and with substrate modulus $k_{s}$, but decreased with nanowire diameter $D$. The system stiffness was larger than with the second-order nonlocal strain gradient model.

The next step in developing the proposed nanowire-substrate model is to consider the effects of geometric nonlinearity (geometric stiffness matrix) in the element. Moreover, this study will be extended to buckling analysis of a nanostructure embedded in an elastic substrate. Finally, the authors hope that the proposed nanowire-substrate model will be useful in the field of nanotechnology.

Acknowledgement: This study was supported by TRF Senior Research Scholar under Grant RTA 6280012. Any opinions expressed in this paper are those of the authors and do not reflect the views of the sponsoring agencies. Special thanks go to the copy-editing service of Research and Development Office, and Assoc. Prof. Dr. Seppo Karrila and Assoc. Prof. Dr. Pattamad Panedpojaman who dedicated his time to provide valuable comments, and gratefully acknowledge the support and assistance received.

\section{APPENDIX A} form:

The homogeneous differential equation of Eq. (29) can be rearranged into the general

$$
\frac{\partial^{6} u_{x}(x)}{\partial x^{6}}+\vartheta_{1} \frac{\partial^{4} u_{x}(x)}{\partial x^{4}}+\vartheta_{2} \frac{\partial^{2} u_{x}(x)}{\partial x^{2}}+\vartheta_{3} u_{x}(x)=0
$$

where the parameters $\vartheta_{1}, \vartheta_{2}$, and $\vartheta_{3}$ are:

$$
\vartheta_{1}=-\frac{1}{\left(e_{0} a\right)^{2}} ; \vartheta_{2}=\frac{\left(E_{x x} A\right)_{e f f}}{E_{x x} A\left(e_{0} a\right)^{4}} ; \text { and } \vartheta_{3}=-\frac{k_{s}}{E_{x x} A\left(e_{0} a\right)^{4}}
$$

For convenience, the parameters in the general solution are defined by:

$$
\alpha=\frac{-\left(\vartheta_{1}^{2} / 3\right)+\vartheta_{2}}{3} ; \beta=\frac{\left(2 \vartheta_{1}^{3} / 27\right)-\left(\vartheta_{1} \vartheta_{2} / 3\right)+\vartheta_{3}}{2} ; \text { and } \Delta=\alpha^{3}+\beta^{2}
$$

There are many solutions of the homogeneous differential equation of Eq. (A1) that depend on sign of the quantity $\Delta=\alpha^{3}+\beta^{2}$. All the possible solution cases are described by Morfidis [52] and Avramidis and Morfidis [53]. They suggest that the most likely solution case I has the quantity $\Delta$ larger than zero $\left(\Delta=\alpha^{3}+\beta^{2}>0\right)$, while in case II $\Delta$ is less than zero $\left(\Delta=\alpha^{3}+\beta^{2}<0\right)$. Thus, these cases are assessed in this study. However, there are other types of solutions when $\Delta$ is equal to zero $\left(\Delta=\alpha^{3}+\beta^{2}=0\right)$. This case is unlikely and almost impossible. Therefore, this study neglected this case.

The general form of the homogeneous solution to Eq. (29) is

$$
u_{x}(x)=\psi_{1}(x) C_{1}+\psi_{2}(x) C_{2}+\psi_{3}(x) C_{3}+\psi_{4}(x) C_{4}+\psi_{5}(x) C_{5}+\psi_{6}(x) C_{6}
$$


For the solution case I $\left(\Delta=\alpha^{3}+\beta^{2}>0\right)$, the analytical solution can be expressed as:

where

$$
\begin{aligned}
& \psi_{1}(x)=e^{R_{1} x} ; \psi_{2}(x)=e^{-R_{1} x} ; \psi_{3}(x)=e^{R x} \cos [Q x] ; \psi_{4}(x)=e^{R x} \sin [Q x] \\
& \psi_{5}(x)=e^{-R x} \cos [Q x] ; \psi_{6}(x)=e^{-R x} \sin [Q x]
\end{aligned}
$$

$$
\begin{aligned}
& R_{1}=\sqrt{\sqrt[3]{-\beta+\sqrt{\Delta}}+\sqrt[3]{-\beta-\sqrt{\Delta}}-\frac{\vartheta_{1}}{3}} ; R=\sqrt{\frac{\left(\sqrt{m^{2}+n^{2}}+m\right)}{2} ; Q=\sqrt{\frac{\left(\sqrt{m^{2}+n^{2}}-m\right)}{2}}} ; \\
& m=-\frac{\left[\sqrt[3]{-\beta+\sqrt{\Delta}}+\sqrt[3]{-\beta-\sqrt{\Delta}}+\left(2 \vartheta_{1} / 3\right)\right]}{2} ; n=\frac{\sqrt{3}[\sqrt[3]{-\beta+\sqrt{\Delta}}-\sqrt[3]{-\beta-\sqrt{\Delta}}]}{2}
\end{aligned}
$$

In case II $\left(\Delta=\alpha^{3}+\beta^{2}<0\right)$, the analytical solution can be written as:

$$
\psi_{1}(x)=e^{R x} ; \psi_{2}(x)=e^{-R x} ; \psi_{3}(x)=e^{R_{1} x} ; \psi_{4}(x)=e^{R_{2} x} ; \psi_{5}(x)=e^{-R_{1} x} ; \psi_{6}(x)=e^{-R_{2} x}
$$

where

$$
\begin{aligned}
& R=\sqrt{2 \sqrt{-\alpha} \cos \left[\frac{\phi}{3}\right]-\frac{\vartheta_{1}}{3}} ; R_{1}=\sqrt{2 \sqrt{-\alpha} \cos \left[\frac{\phi+2 \pi}{3}\right]-\frac{\vartheta_{1}}{3}} ; \\
& R_{2}=\sqrt{2 \sqrt{-\alpha} \cos \left[\frac{\phi+4 \pi}{3}\right]-\frac{\vartheta_{1}}{3}} ; \phi=\cos ^{-1}\left[-\beta / \sqrt{-\alpha^{3}}\right]
\end{aligned}
$$

\section{REFERENCES}

1. Li, X., Bhushan, B., Takashima, K., Baek, C.W., Kim, Y.K., 2003, Mechanical characterization of micro/nanoscale structures for MEMS/NEMS applications using nanoindentation techniques, Ultramicroscopy, 97(1-4), pp. 481-494.

2. Kizuka, T., Takatani, Y., Asaka, K., Yoshizaki, R., 2005, Measurements of the atomistic mechanics of single crystalline silicon wires of nanometer width, Physical Review B, 72(3), 035333.

3. Ganapathi, M., Polit, O., 2018, A nonlocal higher-order model including thickness stretching effect for bending and buckling of curved nanobeams, Applied Mathematical Modelling, 57, pp. 121-141.

4. Demir, C., Mercan, K., Numanoglu, H.M., Civalek, O., 2018, Bending response of nanobeams resting on elastic foundation, Journal of Applied and Computational Mechanics, 4(2), pp. 105-114.

5. Apuzzo, A., Barretta, R., Fabbrocino, F., Faghidian, S.A., Luciano, R., Marotti de Sciarra, F., 2019, Axial and torsional free vibrations of elastic nano-beams by stress-driven two-phase elasticity, Journal of Applied and Computational Mechanics, 5(2), pp. 402-413.

6. Wen, Y.H., Zhu, Z.Z., Zhu, R.Z., 2008, Molecular dynamics study of the mechanical behavior of nickel nanowire: Strain rate effects, Computational Materials Science, 41(4), pp. 553-560.

7. Domekeli, U., Sengul, S., Celtek, M., Canan, C., 2017, The melting mechanism in binary Pd0.25Ni0.75 nanoparticles: molecular dynamics simulations, Philosophical Magazine, 98(5), pp. 371-387.

8. Frink, L.J.D., Salinger, A.G., Sears, M.P., Weinhold, J.D., Frischknecht, A.L., 2002, Numerical challenges in the application of density functional theory to biology and nanotechnology, Journal of Physics Condensed Matter, 14(46), pp. 12167-12187.

9. da Silva, E.Z., Novaes, F.D., da Silva, A.J.R., Fazzio, A., 2004, Theoretical study of the formation, evolution, and breaking of gold nanowires, Physical Review B, 69, 115411.

10. Wang, Q., Varadan, V.K., 2005, Stability analysis of carbon nanotubes via continuum models, Smart Materials and Structures, 14(1), pp. 281-286.

11. Khodabakhshi, P., Reddy, J.N., 2017, A unified beam theory with strain gradient effect and the von Kármán nonlinearity, ZAMM Journal of applied mathematics and mechanics: Zeitschrift für angewandte Mathematik und Mechanik, 97(1), pp. 70-91. 
12. Karličić, D., Murmu, T., Adhikari, S., McCarthy, M., 2016, Non-local structural mechanics, ISTE Ltd and John Wiley \& Sons, Inc.

13. Eringen, A.C., 1972, Nonlocal polar elastic continua, International Journal of Engineering Science, 10(1), pp. 1-16.

14. Eringen, A.C., 1983, On differential equations of nonlocal elasticity and solutions of screw dislocation and surface waves, Journal of Applied Physics, 54(9), pp. 4703-4710.

15. Mindlin, R.D., Tiersten, H.F., 1962, Effects of couple-stresses in linear elasticity, Archive for Rational Mechanics and Analysis, 11, pp. 415-448.

16. Ebrahimi, F., Barati, M.R., 2017, A modified nonlocal couple stress-based beam model for vibration analysis of higher-order FG nanobeams, Mechanics of Advanced Materials and Structures, 25(13), pp. 1121-1132.

17. Sourki, R., Hosseini, S.A., 2017, Coupling effects of nonlocal and modified couple stress theories incorporating surface energy on analytical transverse vibration of a weakened nanobeam, The European Physical Journal Plus, 132, 184.

18. Mindlin, R.D., 1964, Micro-structure in linear elasticity, Archive for Rational Mechanics and Analysis, 16, pp. 51-78.

19. Mindlin, R.D., 1965, On the equations of elastic materials with micro-structure, International Journal of Solids and Structures, 1(1), pp. 73-78.

20. Lam, D.C.C., Yang, F., Chong, A.C.M., Wang, J., Tong, P., 2003, Experiments and theory in strain gradient elasticity, Journal of the Mechanics and Physics of Solids, 51(8), pp. 1477-1508.

21. Thai, H.T., Vo, T.P., Nguyen, T.K., Kim, S.E., 2017, A review of continuum mechanics models for sizedependent analysis of beams and plates, Composite Structures, 177, pp. 196-219.

22. Hu, K.M., Zhang, W.M., Zhong, Z.Y., Peng, Z.K., Meng, G., 2014, Effect of surface layer thickness on buckling and vibration of nonlocal nanowires, Physics Letters A, 378(7-8), pp. 650-654.

23. Khodabakhshi, P., Reddy, J.N., 2015, A unified integro-differential nonlocal model, International Journal of Engineering Science, 95, pp. 60-75.

24. Limkatanyu, S., Sae-Long, W., Horpibulsuk, S., Prachasaree, W., Damrongwiriyanupap, N., 2018, Flexural responses of nanobeams with coupled effects of nonlocality and surface energy, ZAMM Journal of applied mathematics and mechanics: Zeitschrift für angewandte Mathematik und Mechanik, 98(10), pp. 1771-1793.

25. Peddieson, J., Buchanan, G.R., McNitt, R.P., 2003, Application of nonlocal continuum models to nanotechnology, International Journal of Engineering Science, 41(3-5), pp. 305-312.

26. Challamel, N., Wang, C.M., 2008, The small length scale effect for a non-local cantilever beam: a paradox solved, Nanotechnology, 19(34), 345703.

27. Romano, G., Barretta, R., Diaco, M., Marotti de Sciarra, F., 2017, Constitutive boundary conditions and paradoxes in nonlocal elastic nanobeams, International Journal of Mechanical Sciences, 121, pp. 151-156.

28. Altan, B.S., Aifantis, E.C., 1997, On some aspects in the special theory of gradient elasticity, Journal of the Mechanical Behavior of Materials, 8(3), pp. 231-282.

29. Narendar, S., Gopalakrishnan, S., 2010, Ultrasonic wave characteristics of nanorod via nonlocal strain gradient models, Journal of Applied Physics, 107(8), 084312.

30. Askes, H., Suiker, A.S.J., Sluys, L.J., 2002, A classification of higher-order strain-gradient models linear analysis, Archive of Applied Mechanics, 72, pp. 171-188.

31. Zaera, R., Serrano, Ó., Fernández-Sáez, J., 2019, On the consistency of the nonlocal strain gradient elasticity, International Journal of Engineering Science, 138, pp. 65-81.

32. Kong, S., Zhou, S., Nie, Z., Wang, K., 2009, Static and dynamic analysis of micro beams based on strain gradient elasticity theory, International Journal of Engineering Science, 47(4), pp. 487-498.

33. Barretta, R., Marotti de Sciarra, F., 2013, A nonlocal model for carbon nanotubes under axial loads, Advances in Materials Science and Engineering, 360935.

34. Marotti de Sciarra, F., Barretta, R., 2014, A new nonlocal bending model for Euler-Bernoulli nanobeams, Mechanics Research Communications, 62, pp. 25-30.

35. Marotti de Sciarra, F., 2014, Finite element modelling of nonlocal beams, Physica E: Low-dimensional Systems and Nanostructures, 59, pp. 144-149.

36. Marotti de Sciarra, F., Barretta, R., 2014, A gradient model for Timoshenko nanobeams, Physica E: Lowdimensional Systems and Nanostructures, 62, pp. 1-9.

37. Sae-Long, W., Limkatanyu, S., Prachasaree, W., Rungamornrat, J., Sukontasukkul, P., 2020, A thermodynamics-based nonlocal bar-elastic substrate model with inclusion of surface-energy effect, Journal of Nanomaterials, 8276745.

38. Miller, R.E., Shenoy, V.B., 2000, Size-dependent elastic properties of nanosized structural elements, Nanotechnology, 11(3), pp. 139-147.

39. Huang, D.W., 2008, Size-dependent response of ultra-thin films with surface effects, International Journal of Solids and Structures, 45(2), pp. 568-579. 
40. Wang, J., Huang, Z., Duan, H., Yu, S., Feng, X., Wang, G., Zhang, W., Wang, T., 2011, Surface stress effect in mechanics of nanostructured materials, Acta Mechanica Solida Sinica, 24(1), pp. 52-82.

41. Liu, H., Song, H., Feng, X., Yang, J., 2014, Surface effects on the persistence length of nanowires and nanotubes, Theoretical and Applied Mechanics Letters, 4(5), 051009.

42. Gurtin, M.E., Murdoch, I., 1975, A continuum theory of elastic material surface, Archive for Rational Mechanics and Analysis, 57(4), pp. 291-323.

43. Gurtin, M.E., Murdoch, I., 1978, Surface stress in solids, International Journal of Solids and Structures, 14(6), pp. 431-440.

44. Liu, C., Rajapakse, R.K.N.D., 2010, Continuum models incorporating surface energy for static and dynamic response of nanoscale beams, IEEE Transactions on Nanotechnology, 9(4), pp. 422-431.

45. Fu, Y., Zhang, J., Jiang, Y., 2010, Influences of the surface energies on the nonlinear static and dynamic behaviors of nanobeams, Physica E: Low-dimensional Systems and Nanostructures, 42(9), pp. 2268-2273.

46. Fu, G., Zhou, S., Qi, L., 2019, A size-dependent Bernoulli-Euler beam model based on strain gradient elasticity theory incorporating surface effects, ZAMM Journal of applied mathematics and mechanics: Zeitschrift für angewandte Mathematik und Mechanik, 99(6), pp. 1-22.

47. Lim, C.W., He, L.H., 2004, Size-dependent nonlinear response of thin elastic films with nano-scale thickness, International Journal of Mechanical Sciences, 46(11), pp. 1715-1726.

48. Sahmani, S., Aghdam, M.M., Akbarzadeh, A.H., 2016, Size-dependent buckling and postbuckling behavior of piezoelectric cylindrical nanoshells subjected to compression and electrical load, Materials \& Design, 105, pp. 341-351.

49. Limkatanyu, S., Damrongwiriyanupap, N., Prachasaree, W., Sae-Long, W., 2013, Modeling of axially loaded nanowire embedded in elastic substrate media including nonlocal and surface effects, Journal of Nanomaterials, 635428.

50. Limkatanyu, S., Prachasaree, W., Damrongwiriyanupap, N., Kwon, M., 2014, Exact stiffness matrix for nonlocal bars embedded in elastic foundation media: the virtual-force approach, Journal of Engineering Mathematics, 89, pp. 163-176.

51. Klusemann, B., Bargmann, S., Estrin, Y., 2016, Fourth-order strain-gradient phase mixture model for nanocrystalline fcc materials, Modelling and Simulation in Materials Science and Engineering, 24, 085016.

52. Morfidis, K., 2003, Research and development of methods for the modeling of foundation structural elements and soil, $\mathrm{PhD}$ dissertation, Department of Civil Engineering, Aristotle University of Thessaloniki, Greece.

53. Avramidis, I.E., Morfidis, K., 2006, Bending of beams on three-parameter elastic foundation, International Journal of Solids and Structures, 43(2), pp. 357-375.

54. Wolfram, S., 1992, Mathematica reference guide, Addison-Wesley Publishing Company, Redwood City, California.

55. Winkler, E., 1867, Die Lehre von der und Festigkeit, Dominicus, Prag.

56. Tonti, E., 1976, The reason for analogies between physical theories, Applied Mathematical Modelling, 1(1), pp. 37-50.

57. Juntarasaid, C., Pulngern, T., Chucheepsakul, S., 2012, Bending and buckling of nanowires including the effects of surface stress and nonlocal elasticity, Physica E: Low-dimensional Systems and Nanostructures, 46, pp. 68-76.

58. He, J., Lilley, C.M., 2008, Surface effect on the elastic behavior of static bending nanowires, Nano Letters, 8(7), pp. 1798-1802.

59. Shenoy, V.B., 2006, Atomistic calculations of elastic properties of metallic fcc crystal surfaces, Physical Review B, 74(14), 149901.

60. Liew, K.M., He, X.Q., Kitipornchai, S., 2006, Predicting nanovibration of multi-layer grapheme sheets embedded in an elastic matrix, Acta Materialia, 54(16), pp. 4229-4236.

61. Assadi, A., Farshi, B., Alinia-Ziazi, A., 2010, Size dependent dynamic analysis of nanoplates, Journal of Applied Physics, 107(12), 124310.

62. Jiang, L.Y., Yan, Z., 2010, Timoshenko beam model for static bending of nanowires with surface effects, Physica E: Low-dimensional Systems and Nanostructures, 42(9), pp. 2274-2279.

63. Limkatanyu, S., Kuntiyawichai, K., Spacone, E., Kwon, M., 2012, Natural stiffness matrix for beams on Winkler foundation: exact force-based derivation, Structural Engineering and Mechanics, 42(1), pp. 39-53.

64. Khajeansari, A., Baradaran, G.H., Yvonnet, J., 2012, An explicit solution for bending of nanowires lying on Winkler-Pasternak elastic substrate medium based on the Euler-Bernoulli beam theory, International Journal of Engineering Science, 52, pp. 115-128. 